\title{
PROPAGATION AND INTERACTION OF ULTRASHORT ELECTROMAGNETIC PULSES IN NONLINEAR MEDIA WITH A QUADRATIC-CUBIC NONLINEARITY
}

\author{
Elena V. Kazantseva ${ }^{a}$ 円 and Andrey I. Maimistov ${ }^{a}$ [ \\ Boris A. Malomed ${ }^{b}$ \\ ${ }^{a}$ Department of Solid State Physics, Moscow Engineering Physics Institute, \\ Moscow, 115409, Russia \\ ${ }^{b}$ Department of Interdisciplinary Studies, Faculty of Engineering, Tel Aviv \\ University, Tel Aviv 69978, Israel
}

\footnotetext{
${ }^{1}$ electronic addres: e-lena@pico.mephi.ru

${ }^{2}$ electronic address: maimistov@pico.mephi.ru

${ }^{3}$ electronic address malomed@eng.tau.ac.il
} 


\begin{abstract}
Propagation of extremely short unipolar pulses of electromagnetic field ("videopulses") is considered in the framework of a model in which the material medium is represented by anharmonic oscillators (approximating bound electrons) with quadratic and cubic nonlinearities. Two families of exact analytical solutions (with positive or negative polarity) are found for the moving solitary pulses. Direct simulations demonstrate that the pulses are very robust against perturbations. Two unipolar pulses collide nearly elastically, while collisions between pulses with opposite polarities and a small relative velocity are inelastic, leading to emission of radiation and generation of a small-amplitude additional pulse.
\end{abstract}

PACS number: 42.65.Tg 


\section{INTRODUCTION}

Very short nonlinear pulses of the electromagnetic field, which contain few optical cycles [1, 2, 3, 4, 5], or even of half a cycle [6], have recently attracted a great deal of attention. They are referred to as ultrashort pulses (USP), or videopulses [1, 1, 5. From the viewpoint of applications, the interest to these pulses is stipulated by a perspective of a drastic increase in the rates of data transmission and procession. One of possibilities for generation of USPs is compression of a preliminarily phase-modulated pulse. The phase modulation can be induced by the Kerr (cubic) nonlinearity [7]. For example, pulses with the duration $\tau_{p}=4.5 \mathrm{fs}$, consisting of two optical cycles with the frequencies belonging to the visible range, and with $\tau_{p}=40 \mathrm{fs}$, containing just one optical cycle at the frequency of the $\mathrm{CO}_{2}$ laser, were reported in Ref. [8].

If the width of a pulse is much larger than the period of the optical cycle, the pulse can be naturally described by means of its slowly varying envelope, whose evolution is governed by an effective parabolic (nonlinear Schrödinger) equation [7]. However, this approximation cannot be applied to USPs, which calls for development of other theoretical models admitting a relatively simple description of the propagation of extremely short pulses.

The existing models that can be used for the description of USPs fall into two classes, resonant and nonresonant ones. A resonant medium is modeled by an ensemble of atoms with discrete energy levels. The field frequency being close to a frequency of an atomic transition, only the (nearly) resonant component of the field is taken into account. In particular, the simplest model of the self-induced transparency assumes a medium consisting of twolevel atoms resonantly coupled to the field. In the case of multi-level atoms, it may be necessary to consider several monochromatic components of the field with different frequencies.

In the works [9, 10, 11], USP solutions to a closed system of the MaxwellBloch equations have been found analytically without assuming a separation into the carrier wave and envelope. Generally, the Maxwell's equations admit the propagation of electromagnetic waves in both directions. If, however, the nonlinear contribution into the medium's polarization is small, the unidirectional wave propagation may be assumed. This approximation admits reducing the wave equation to the first-order one without any assumption about the shape of the waves.

The nonlinear dynamics of the medium driven by the electromagnetic 
filed is frequently modeled in terms of anharmonic oscillators. In particular, the propagation of a linearly polarized USP was considered in Refs. [2, 12] in the framework of the material model based on the Duffing oscillators, the nonlinear response of the medium being cubic. The propagation of a linearly polarized USP in a dispersive medium was modeled by means of quadratically nonlinear oscillators in Ref. [四] (without dispersion, this was done in Ref. 13]).

In all these works, the oscillator represents a response of the high-frequency electron degree of freedom to the electromagnetic field. Since the USP spectrum is concentrated at low frequencies, ion oscillations may also give a considerable contribution to the full polarization of the medium. Nevertheless, we will neglect the ion component in the material response (the propagation of femtosecond pulses in a medium with the nonlinearity determined by both electronic and ionic (Raman-scattering) degrees of freedom was considered in Ref. [14]).

An objective of the present work is to study the unidirectional propagation and interactions of linearly polarized USPs in a nonlinear dispersive medium modeled by an anharmonic oscillator combining quadratic and cubic nonlinearities. As is well known, in the case when the oscillator is quasiharmonic, the quadratic and cubic nonlinear terms produce effects of the same order of magnitude [15], that is why it is natural to consider a mixed model of this type. It is also relevant to mention that modeling dynamics of the broad (rather than ultrashort) optical solitons in a medium with competing quadratic and cubic nonlinearities has recently attracted considerable attention, see, e.g., the works [16] and references therein.

The rest of the paper is structured as follows. The model is derived in section 2. Two families of moving USP solutions with positive and negative polarities are found analytically in section 3 (only one family survives in the limit of a vanishing cubic nonlinearity). Stability of the pulses and their collisions (for both signs of the relative polarity of the colliding pulses) are investigated in section 4 by means of direct simulations.

\section{THE MODEL}

The one-dimensional propagation of the electromagnetic waves in a nonlinear medium is governed by the wave equation, 


$$
\frac{\partial^{2} E}{\partial z^{2}}-\frac{1}{c^{2}} \frac{\partial^{2} E}{\partial t^{2}}=\frac{4 \pi}{c^{2}} \frac{\partial^{2} P}{\partial t^{2}}
$$

where $P$ is the polarization of the medium. In the framework of the usual unidirectional approximation [17, 18], Eq. (11) can be reduced to the firstorder equation,

$$
\frac{\partial E}{\partial z}+\frac{1}{c} \frac{\partial E}{\partial t}=-\frac{2 \pi}{c} \frac{\partial P}{\partial t}
$$

We adopt a simple anharmonic-oscillator model for the medium, which is a frequently used approximation [19] (see also [20, 21]). If $X$ as the displacement of an electron from its equilibrium position, the motion equation (which neglects friction) can be written as

$$
\frac{\partial^{2} X}{\partial t^{2}}+\omega_{0}^{2} X-\kappa_{2} X^{2}+\kappa_{3} X^{3}=\mathfrak{L} \frac{e}{m} E(z, t),
$$

where $\omega_{0}$ is an eigenfrequency of the oscillator, while $\kappa_{2}$ and $\kappa_{3}$ are anharmonicity coefficients. The term on the right-hand side of Eq. (3) represents the force exerted on the electron by the electromagnetic field, where $\mathfrak{L}=(\varepsilon+2) / 3$ is the Lorentz factor, $e$ is the electron's electric charge, and $m$ is its mass. However, it is possible to absorb $\mathfrak{L}$ into an effective mass $m_{\text {eff }} \equiv m / \mathfrak{L}$. Hereafter, we will use $m$ as a symbol for this effective mass. Finally, the dynamical variable $X$ is related to the medium's polarization, $P=n_{A} e X$, where $n_{A}$ is the density of the oscillators (atoms).

We define rescaled variables,

$$
\zeta \equiv z / l, \tau \equiv \omega_{0}(t-z / c), \mathcal{E} \equiv E / A_{0}, \text { and } q \equiv X / X_{0}
$$

where $A_{0}=m \omega_{0}^{4} / e\left|\kappa_{2}\right|, \quad X_{0}=\omega_{0}^{2} /\left|\kappa_{2}\right|, \quad 1 / l=2 \pi n_{A} e^{2} /\left(m c \omega_{0}\right)=\omega_{p}^{2} / 2 \omega_{0} c$, and

$$
\omega_{p}=\left(4 \pi n_{A} e^{2} / m\right)^{-1 / 2}
$$

is the plasma frequency. In terms of the rescaled variables, Eqs. (2) and (3) take the form

$$
\frac{\partial \mathcal{E}}{\partial \zeta}=-\frac{\partial q}{\partial \tau}, \quad \frac{\partial^{2} q}{\partial \tau^{2}}+q-q^{2}+2 \mu q^{3}=\mathcal{E},
$$

with the single remaining parameter $\mu=\left(\kappa_{3} \omega_{0}^{2} / 2 \kappa_{2}^{2}\right)$. Equations (6) furnish a final form of the model. 
To estimate the magnitude of parameter $\mu$, we consider Morse potential

$$
U_{M}(X)=U_{0}\left[\exp \left(-2 X / r_{0}\right)-2 \exp \left(-X / r_{0}\right)\right],
$$

where $r_{0}$ is an atomic characteristic size and $U_{0}$ is a ionization potential. The expansion of this potential in terms of a power series in $X$ leads to $\omega_{0}^{2}=2 U_{0} / m r_{0}^{2},\left|\kappa_{2}\right|=3 U_{0} / m r_{0}^{3}, \kappa_{3}=7 U_{0} / 3 m r_{0}^{4}$. Thus $\mu=7 / 27 \approx 0.259$.

Consider now Kepler's potential with Coulomb long-range attraction:

$$
U_{K}(X)=U_{0}\left[\left(\frac{r_{0}}{r_{0}+X}\right)^{2}-2\left(\frac{r_{0}}{r_{0}+X}\right)\right],
$$

where $r_{0}$ corresponds to equilibrium position. In this case we have $\omega_{0}^{2}=$ $2 U_{0} / m r_{0}^{2},\left|\kappa_{2}\right|=6 U_{0} / m r_{0}^{3}, \kappa_{3}=12 U_{0} / m r_{0}^{4}$, and $\mu=1 / 3$. In following numerical simulation of the steady state pulses propagation about the same magnitude of parameter $\mu$ will be used.

\section{DYNAMICAL INVARIANTS}

It is worthy to note that the system of equations (6) can be derived as the Euler-Lagrange equations from the action functional

$$
S=\int \mathcal{L}[q, \phi] d x d t
$$

where the Lagrangian density is

$$
\mathcal{L}=\frac{1}{2} \frac{\partial \phi}{\partial x} \frac{\partial \phi}{\partial t}+\frac{1}{2}\left(\frac{\partial q}{\partial x}\right)^{2}-\frac{1}{2} q^{2}+\frac{1}{2} q^{3}-\frac{\mu}{2} q^{4}+q \frac{\partial \phi}{\partial x},
$$

and the independent variables were temporarily (up to the end of this section) redenoted as $\zeta \rightarrow t, \tau \rightarrow x$, in order to have the notation similar to that in the classical field theory (a definition of $\phi$ is given below).

Application of the variational procedure to the action $S$ yields equations

$$
\frac{\partial^{2} \phi}{\partial t \partial x}+\frac{\partial^{2} q}{\partial x^{2}}=0, \quad \frac{\partial^{2} q}{\partial t^{2}}+q-q^{2}+2 \mu q^{3}=\frac{\partial \phi}{\partial x} .
$$

Identifying $\phi$ as a potential for the fields $q$ and $\mathcal{E}$, so that $q \equiv-\partial \phi / \partial t$ and $\mathcal{E} \equiv \partial \phi / \partial x$, makes these equations identical to Eqs. (6), which can be further transformed into a single equation 


$$
\frac{\partial q}{\partial t}+\frac{\partial q}{\partial x}-2 q \frac{\partial q}{\partial t}+6 \mu q^{2} \frac{\partial q}{\partial t}+\frac{\partial^{3} q}{\partial t \partial x^{2}}=0
$$

Note that Eq. (9) may be regarded as a continuity equation,

$$
\frac{\partial}{\partial t}\left(q-q^{2}+2 \mu q^{3}+\frac{\partial^{2} q}{\partial x^{2}}\right)+\frac{\partial q}{\partial x}=0 .
$$

Integration of Eq. (10) in $x$ leads to

$$
\frac{\partial}{\partial t} \int_{-\infty}^{+\infty}\left(q-q^{2}+2 \mu q^{3}\right) d x+\frac{\partial}{\partial t}\left(\left.\frac{\partial q}{\partial x}\right|_{-\infty} ^{+\infty}\right)+\left.q\right|_{-\infty} ^{+\infty}=0
$$

Assuming zero boundary conditions for $q$ and its derivatives at $x \rightarrow \pm \infty$, the latter relation implies the conservation of a dynamical invariant,

$$
I_{1}=\int_{-\infty}^{+\infty}\left(q-q^{2}+2 \mu q^{3}\right) d x
$$

By taking into account the second equation from Eqs. (6) in the form

$$
\frac{\partial^{2} q}{\partial x^{2}}+q-q^{2}+2 \mu q^{3}=\mathcal{E}
$$

one can find that $I_{1}$ is the "area of the pulse",

$$
I_{1}=\int_{-\infty}^{+\infty} \mathcal{E} d x
$$

To find another dynamical invariant, we multiply Eq. (9) by $q$ and transform the resulting relation into a form

$$
\frac{\partial}{\partial t}\left[\frac{1}{2} q^{2}-\frac{2}{3} q^{3}+\frac{3 \mu}{2} q^{4}-\frac{1}{2}\left(\frac{\partial q}{\partial x}\right)^{2}\right]+\frac{\partial}{\partial x}\left(\frac{1}{2} q^{2}+q \frac{\partial^{2} q}{\partial x \partial t}\right)=0 .
$$

Thus, a new continuity equation is obtained, giving rise to a new dynamical invariant, 


$$
I_{2}=\int_{-\infty}^{+\infty}\left[\frac{1}{2} q^{2}-\frac{2}{3} q^{3}+\frac{3 \mu}{2} q^{4}-\frac{1}{2}\left(\frac{\partial q}{\partial x}\right)^{2}\right] d x
$$

Let us now get back to the conserved density in the continuity equation (10),

$$
Q \equiv q-q^{2}+2 \mu q^{3}+\frac{\partial^{2} q}{\partial x^{2}}
$$

Multiplying both sides of Eq. (10) by $Q$, we obtain

$$
\frac{\partial}{\partial t}\left(\frac{1}{2} Q^{2}\right)+Q \frac{\partial q}{\partial x}=0
$$

This immediately leads to a continuity equation

$$
\frac{\partial}{\partial t}\left(\frac{1}{2} Q^{2}\right)+\frac{\partial}{\partial x}\left[\frac{1}{2} q^{2}-\frac{1}{3} q^{3}+\frac{\mu}{2} q^{4}+\frac{1}{2}\left(\frac{\partial q}{\partial x}\right)^{2}\right]=0
$$

and the third dynamical invariant,

$$
I_{3}=\frac{1}{2} \int_{-\infty}^{+\infty}\left(q^{2}-q^{3}+2 \mu q^{4}+\frac{\partial^{2} q}{\partial x^{2}}\right)^{2} d x
$$

Taking into account the relation (12), this integral may be interpreted as a "pulse energy",

$$
I_{3}=\frac{1}{2} \int_{-\infty}^{+\infty} \mathcal{E}^{2} d x
$$

To find the interpretation of the dynamical invariant given by Eq. (15), we resort to the Lagrangian density (7). The density of the canonical Hamiltonian for this dynamical system can be obtained from $\mathcal{L}$ by means of the standard Legendre transformation,

$$
\mathcal{H}=\frac{\partial \mathcal{L}}{\partial \phi_{, t}} \phi_{, t}-\frac{\partial \mathcal{L}}{\partial q_{, t}} q_{, t}-\mathcal{L}=-\frac{1}{2}\left(\frac{\partial q}{\partial x}\right)^{2}+\frac{1}{2} q^{2}-\frac{1}{3} q^{3}+\frac{\mu}{2} q^{4}-q \mathcal{E} .
$$

The variable $\mathcal{E}$ can be eliminated from it, using Eq. (12), so that 


$$
\mathcal{H}=-\frac{\partial}{\partial x}\left(q \frac{\partial q}{\partial x}\right)+\frac{1}{2}\left(\frac{\partial q}{\partial x}\right)^{2}-\frac{1}{2} q^{2}+\frac{2}{3} q^{3}-\frac{3 \mu}{2} q^{4}
$$

Omitting the full derivative, the Hamiltonian corresponding to the density (19) takes the form

$$
H=\int_{-\infty}^{+\infty}\left[\frac{1}{2}\left(\frac{\partial q}{\partial x}\right)^{2}-\frac{1}{2} q^{2}+\frac{2}{3} q^{3}-\frac{3 \mu}{2} q^{4}\right] d x \equiv-I_{2}
$$

cf. Eq. (15). Thus, the dynamical invariant $-I_{2}$ is nothing else but the canonical Hamiltonian of the system under consideration.

\section{ANALYTICAL SOLUTIONS FOR THE UL- TRASHORT PULSES}

It seems plausible that the system of Eqs. (6) is not an integrable one. Nevertheless, some exact analytical solutions, describing the propagation of USPs, can be found. To this end, one assumes that $\mathcal{E}$ and $q$ depend on a single variable,

$$
\eta \equiv \tau-\zeta / \alpha=\omega_{0}(t-z / V),
$$

with some constant $\alpha$. An expression for the velocity $V$ of a steadily moving pulse then follows from Eq. (4),

$$
\frac{1}{V}=\frac{1}{c}\left[1+\frac{1}{2 \alpha}\left(\frac{\omega_{p}}{\omega_{0}}\right)^{2}\right]
$$

$\omega_{p}$ being the plasma frequency defined by Eq. (5). The first equation of the system (6) can be integrated to yield

$$
\mathcal{E}=\alpha q
$$

Next, the second equation from the system (6) takes the form

$$
\frac{d^{2} q}{d \eta^{2}}-(\alpha-1) q-q^{2}+2 \mu q^{3}=0
$$

This equation can be integrated once, 


$$
\left(\frac{d q}{d \eta}\right)^{2}-(\alpha-1) q^{2}-\frac{2}{3} q^{3}+\mu q^{4}=\text { const }
$$

As we are interested in solitary-wave solutions, it is necessary to set const $=$ 0 , so that (24) yields

$$
\frac{d q}{d \eta}= \pm q \sqrt{(\alpha-1)+\frac{2}{3} q-\mu q^{2}},
$$

A substitution $q \equiv 1 / y$ transforms Eq. (25) into

$$
\frac{d y}{d \eta}= \pm \sqrt{(\alpha-1)\left[\left(y+\frac{1}{3(\alpha-1)}\right)^{2}-\frac{1+9 \mu(\alpha-1)}{9(\alpha-1)^{2}}\right]} .
$$

A new dependent variable $\xi$ will be used, defined by

$$
y+\frac{1}{3(\alpha-1)} \equiv \sigma\left(\frac{1+9 \mu(\alpha-1)}{9(\alpha-1)^{2}}\right)^{1 / 2} \xi(\eta),
$$

with $\sigma= \pm 1$. In terms of $\xi$, Eq. (26) is

$$
\sigma \frac{d \xi}{d \eta}= \pm \sqrt{(\alpha-1)\left(\xi^{2}-1\right)}
$$

from where one finds $\xi(\eta)=\cosh (\sqrt{(\alpha-1)} \eta)$.

Thus, we have obtained a family of exact solutions parameterized by the continuous positive parameter $(\alpha-1)$ and discrete one $\sigma= \pm 1$,

$$
\begin{gathered}
q^{(+)}(\eta ; \alpha)=\frac{3(\alpha-1)}{\sqrt{1+9(\alpha-1) \mu} \cosh (\sqrt{(\alpha-1)} \eta)-1}, \\
q^{(-)}(\eta ; \alpha)=-\frac{3(\alpha-1)}{\sqrt{1+9(\alpha-1) \mu} \cosh (\sqrt{(\alpha-1)} \eta)+1}
\end{gathered}
$$

the superscript standing for $\sigma$.

The pulses represented by the solutions (27) and (28) have different polarities. In the limit $\mu \rightarrow 0$, corresponding to the model with a purely quadratic nonlinearity, Eq. (27) goes over into a singular solution,

$$
q_{\text {quadr }}^{(+)}(\alpha ; \eta)=\frac{3(\alpha-1)}{2 \sinh ^{2}(\sqrt{(\alpha-1)} \eta / 2)},
$$


while Eq. (28) yields a nonsingular pulse in the same limit, which was already found in Ref. [23,

$$
q_{\text {quadr }}^{(-)}(\alpha ; \eta)=-\frac{3(\alpha-1)}{2 \cosh ^{2}(\sqrt{(\alpha-1)} \eta / 2)} .
$$

Thus, in compliance with the results of Ref. [23], only one family of nonsingular pulses exists in the case of the purely quadratic nonlinearity.

By using Eq. (22), we retrieve an expression for the electromagnetic field corresponding to the solutions (27) and (28),

$$
\begin{gathered}
\mathcal{E}^{(+)}(\alpha ; \eta)=\frac{3 \alpha(\alpha-1)}{\sqrt{1+9(\alpha-1) \mu} \cosh (\sqrt{(\alpha-1)} \eta)-1} \\
\mathcal{E}^{(-)}(\alpha ; \eta)=-\frac{3 \alpha(\alpha-1)}{\sqrt{1+9(\alpha-1) \mu} \cosh (\sqrt{(\alpha-1)} \eta)+1}
\end{gathered}
$$

We stress that the velocities of two pulses which have the opposite polarities but the same value of $(\alpha-1)$ are equal, since they are determined by Eq. (21) and depend only on $\alpha$.

\section{NUMERICAL SIMULATION OF PROP- AGATION AND COLLISIONS OF THE PULSES}

Although the pulse solutions have been obtained in the exact form, their stability and interactions should be studied by means of numerical simulations. To simulate the system (6), it is convenient to transform it into the following form:

$$
\begin{gathered}
\frac{\partial \mathcal{E}}{\partial \zeta}=-p, \\
\frac{\partial q}{\partial \tau}=p, \quad \frac{\partial p}{\partial \tau}=\mathcal{E}-q+q^{2}-2 \mu q^{3} .
\end{gathered}
$$

Since Eq. (31) contains only the derivative with respect to $\zeta$, and the derivatives with respect to $\tau$ are present only in Eqs. (32), one can use any technique of numerical integration of ordinary differential equations. For solving 
Eqs. (32), we employed the prediction-correction method. The fourth-order Runge-Kutta routine was used for integrating Eq. (31). As the initial conditions, we took the analytical solutions given by Eqs. (27) and (29), or (28) and (30), at $\zeta=0$ (with regard to Eq. (20)). The boundary conditions are $q(\zeta, \tau)=p(\zeta, \tau)=0$ at $|\tau| \longrightarrow \infty$.

By using the Morse $U_{M}(X)$ and Kepler $U_{K}(X)$ potentials one can estimate the normalized amplitudes $A_{0}$ and $X_{0}$. For Morse potential $A_{0}=$ $(4 / 3)\left(U_{0} / e r_{0}\right), X_{0}=(2 / 3) r_{0}$, and for Kepler's potential we have $A_{0}=(2 / 3)\left(U_{0} / e r_{0}\right), X_{0}=$ $(1 / 3) r_{0}$. In both case $\left(U_{0} / e r_{0}\right)$ can be identified with atomic field 21. Consequently, the peak amplitude of the steady state pulse must be not exceed $\left(U_{0} / e r_{0}\right)$. Furthermore, the anharmonic-oscillator model proposes that the magnitude of $q$ is less than one. It leads to the inequality $(\alpha-1)<1$. However, in order to obtain the descriptive illustrations of the numerical results the parameter $\alpha$ have been taken both from the interval $1.01<\alpha<1.1$ and from the interval $2<\alpha<8$.

A typical numerical result, with $\alpha=7.25$ and $\mu=2 / 9$, is displayed in Fig.1, which demonstrates stable propagation of the pulse, at least up to $\zeta=200$. To further test the robustness of USPs, we added to the initial configuration perturbations in the form of a quasi-harmonic or biharmonic wave with amplitudes $\leq 0.1$ of the pulse's amplitude, and with different frequencies. As a typical example, Fig. 2 demonstrates evolution of the pulse with $\alpha=5$ and $\mu=1 / 3$, with the initially added perturbation in the form of a long quasi-monochromatic packet filled by the wave $\delta \mathcal{E}(\tau)=\delta \mathcal{E}_{0} \sin (15 \tau)$. As is seen in from Fig. 2, USP is not scathed by the perturbation. With the increase of the amplitude of the quasi-harmonic perturbation, we observed no conspicuous change of USP. Simulations of the pulses evolution in the presence of a biharmonic (two-frequency) perturbation (not shown here) showed that the increase of the amplitudes of the biharmonic perturbation results in a small change of the value of the parameter $\alpha$ corresponding to the finally established USP. A similar effect was generated by an initial perturbation in the form of an additional rectangular pulse superimposed on USP, see an example in Fig. 3. The increase of the perturbation's amplitude leads to an increase of the final value of the USP's amplitude and decrease of its velocity.

Simulations of interactions between pulses with widely different values of the parameter $\alpha$ (i.e., velocities) have shown that their collisions are quasielastic, irrespective of the polarities of the colliding pulses: after passing through each other, the pulses retrieve the same shapes and velocities as they had before the collision, the only result being a shift of their centers. How- 
ever, the character of the interaction between pulses with equal and opposite polarities becomes different with the decrease of their relative velocity. In the case of equal polarities, the pulses with a small relative velocity demonstrate strong mutual repulsion: the distance between them attains a minimum, and then they start to separate again, so that they never completely overlap. Strong energy exchange between the pulses takes place around the point where they attain the minimum separation. The energy exchange gives rise to mutual interconversion of the two pulses, so that after the collision they, effectively, swap their positions. This picture, a typical example of which is displayed in Fig. 4, is quite similar to the classical description of collisions between solitons in the Korteweg - de Vries equation [22].

Collisions between pulses with opposite polarities and close velocities are found to be more inelastic in comparison with the unipolar pulses. In this case, dispersive wave packets with a considerable amplitude are generated, see an example in Fig. 5. Moreover, a new small-amplitude pulse with the negative polarity is also generated by the inelastic collision shown in Fig. 5.

Another noteworthy feature revealed by the simulations is that the results of the collisions are very different depending on which solitons (faster or slower ones) have initially positive and negative polarities. This feature is obvious from the comparison of Figs. 5 and 6 , which differ by the polarity reversal of the initial state. As is seen, in the latter case the inelasticity is much weaker, and, in particular, no additional pulse is generated.

To further illustrate the inelasticity of the collisions, in Fig. 7 we display the shapes of the fields before and after collisions that were shown in Figs. 5 and 6 . In the former case (Fig. 7a), the amplitudes of the positive- and negative-polarity pulses decreases and increases, respectively, as a result of the collision. In the latter case (Fig. 7b), the result is the opposite. From here, we conclude that, in the course of the collision, the energy is always transferred to a more powerful pulse (the one with a larger value of $\alpha$ ).

We also explored interactions between two pulses with equal values of $\alpha$ (hence, they have zero relative velocity), initially placed at various distances from each other. In Fig.8, solid lines show trajectories of the motion of centers of the interacting pulses with different polarities, and dotted lines show the same for interacting unipolar pulses. In the case shown in this figure, the initial distance between the pulses is three half-widths of a steady-state pulse. At such a distance, tails of the pulses overlap considerably, giving rise to energy transfer from one pulse to the other (irrespective of the polarities, no visible interaction between pulses takes place if the initial distances is 
increased to five half-widths). As a result, the pulses cease to be identical and start to separate (the pulse with a larger amplitude is moving slower). Eventually, the pulses assume permanent (but different) shapes after the separation.

In the case of two unipolar pulses, energy is gained by the rear one, therefore the trajectories of the pulses do not intersect in this case, see Fig. 8. On the contrary, in the case of opposite polarities the front positive-polarity pulse gains energy as a result of the interaction, while the absolute value of the amplitude of the negative-polarity rear pulse decreases and its velocity increases, hence the trajectories of the two pulses intersect. After their collision, there appears a new small-amplitude pulse with negative polarity (its trajectory is not shown in Fig. 8), cf. Figs. 5 and 7a.

We stress that we have never observed formation of a bound state of pulses with equal or opposite polarities as a result of their interaction, nor was it possible to find bound states in any other way.

To conclude this section, it is relevant to compare the present model with some others. Indeed, after transforming the system into the single equation (9), replacing $\zeta$ and $\tau$ by $t$ and $x, q$ by $-u$, and setting $\mu=0$ one obtains

$$
\frac{\partial u}{\partial t}+\frac{\partial u}{\partial x}+2 u \frac{\partial u}{\partial t}+\frac{\partial^{3} u}{\partial t \partial x^{2}}=0
$$

which resembles to the so-called regularized long-wave equation [24, 25],

$$
\frac{\partial u}{\partial t}+\frac{\partial u}{\partial x}+2 u \frac{\partial u}{\partial x}-\frac{\partial^{3} u}{\partial t \partial x^{2}}=0 .
$$

While Eqs. (33) and (34) have similar traveling-pulse solutions, the equations are not equivalent, hence they may produce very different dynamical effects.

\section{CONCLUSION}

We have introduced and analyzed a model for the propagation of ultrashort unipolar pulses of electromagnetic field in a material medium represented by anharmonic oscillators with quadratic and cubic nonlinearities. Two families of exact analytical solutions, with positive and negative polarities, have been found for moving solitary pulses. Direct simulations have demonstrated strong stability of the pulses against various perturbations. Collisions between the pulses were also simulated in detail, showing that they interact 
nearly elastically, irrespective of the pulses' relative polarity, unless their relative velocity is very small. If the case of the small relative velocity, collisions are inelastic, resulting in generation of radiation and a new small-amplitude soliton.

\section{Acknowledgment}

We are grateful to S.V. Sazonov and S.A.Kozlov for valuable discussions. A part of this work was supported by INTAS (European Union) under the grant No. 96-0339. 


\section{References}

[1] O. B. Dubrovskaya and A. P. Sukhorukov, Izv. RAN (Proceedings of the Russian Academy of Sciences), ser. Phys. 56 (1992) 184.

[2] A.E. Kaplan, Phys. Rev. Lett. 73 (1994) 1243.

[3] A.E. Kaplan and P.L. Shkolnikov, Phys. Rev. Lett. 75 (1995) 2316.

[4] S.V. Sazonov and E.V. Trifonov, J. Phys. B 27 (1994) L7.

[5] S.V. Sazonov and L.S. Yakupova, J. Phys. B 27 (1994) 369.

[6] A.E. Kaplan, S.F. Straub, and P.L. Shkolnikov, Opt. Lett. 22 (1997) 405.

[7] G.P. Agrawal. Nonlinear Fiber Optics (Academic Press: San Diego, 1995).

[8] I. Babzien , I.V. Pogorelsky, I. Ben-Zim I., J. Skaritka, and I.K. Meskovsky, Terawatt picosecond CO2 lasers and applications, in: Conference on Lasers and Electro-Optics, Technical Digest, p. 388 (Opt. Soc. Am.: Baltimore, 1997).

[9] R.K. Bullough, in: Interaction of Radiation with Condensed Matter, vol. 1, paper IAEA-SMR-20/51, p.381-469 (International Atomic Energy Agency: Vienna, 1977).

[10] K. Akimoto, J. Phys. Soc. Jpn. 65 (1996) 2020.

[11] A.V. Andreev, Phys. Lett. A 179 (1993) 23.

[12] A.I. Maimistov and S.O. Elyutin, J. Mod. Opt. 39 (1992) 2201.

[13] A.A. Akopyan and D.L. Oganesyan, Quant. Electron. 25 (1998) 954 (in Russian).

[14] S.A. Kozlov and S.V. Sazonov, JETP 84 (1997) 221.

[15] L.D. Landau and E.M. Lifshits. Mechanics (Nauka Publishers: Moscow, 1973). 
[16] S. Trillo, A.V. Buryak, and Y.S. Kivshar, Opt. Comm. 122 (1996) 200; O. Bang, Y.S. Kivshar, and A.V. Buryak, Opt. Lett. 22 (1997) 1680; T.J. Alexander, A.V. Buryak, and Y.S. Kivshar, Opt. Lett. 23 (1998) 670; J. Yang, B.A. Malomed, and D.J. Kaup, Phys. Rev. Lett. 83 (1999) 1958 .

[17] R.K. Bullough, P.M. Jack, P.W. Kitchenside, and R. Saunders, Phys. Scripta, 20 (1979) 364.

[18] J.L. Eilbeck, J.D. Gibbon, P.J. Caudrey, and R.K. Bullough, J. Phys. A 6 (1973) 1337.

[19] N. Bloembergen. Nonlinear Optics (Benjamin: New York, 1965).

[20] A. Yariv. Quantum Electronics (John Wiley and Sons, Inc.: New York and London, 1967).

[21] P.N. Butcher, and D. Cotter. The Elements of Nonlinear Optics (Cambridge University Press, 1990).

[22] M.J. Ablowitz, and H. Segur. Solitons and the Inverse Scattering Transform (SIAM, Philadelphia, 1981) .

[23] E.V. Kazantseva and A.I. Maimistov, Phys. Lett. A 263 (1999) 434.

[24] L. Courtency, and J.A. Tjon, Phys. Lett. A 73 (1979) 275.

[25] A.R. Santarelli, Nuovo Cimento B 46 (1978) 179. 


\section{FIGURE CAPTIONS}

Fig. 1. Numerically simulated propagation of the positive-polarity (a) and negative-polarity (b) ultrashort pulses predicted by the analytical solutions (27), (29) and (28), (30) with $\alpha=7.25$ and $\mu=2 / 9$.

Fig. 2. Numerically simulated evolution of the pulse with $\alpha=5$ and $\mu=1 / 3$, with a superimposed perturbation in the form of a quasi-harmonic wave packet.

Fig. 3. The same as in Fig. 2, with an initial perturbation in the form of an additional rectangular pulse.

Fig. 4. An example of a numerically simulated collision between two unipolar pulses with close velocities, corresponding to $\alpha=5$ and $\alpha=4.61$, at $\mu=1 / 3$.

Fig. 5. Collision between pulses with the negative and positive polarities in the case of $\mu=1 / 3$. The pulse of negative polarity relates to $\alpha=5$ and pulse with positive polarity corresponds with $\alpha=3.89$.

Fig. 6. Collision of pulses with the same values of $\alpha$ and $\mu$ as in a fig. 5, but with reversed polarities.

Fig. 7. The shapes of the pulses before and after the collisions, the panels (a) and (b) pertaining to the two cases shown in Figs. 5 and 6.

Fig. 8. Trajectories of the motion of two interacting soliton with exactly equal initial velocities (equal values of $\alpha$ ). The solid and dotted pairs of lines pertain, respectively, to the solitons with opposite and equal polarities. 
This figure "1a.jpg" is available in "jpg" format from: http://arxiv.org/ps/nlin/0010050v1 
This figure "1b.jpg" is available in "jpg" format from: http://arxiv.org/ps/nlin/0010050v1 
This figure "2.jpg" is available in "jpg" format from: http://arxiv.org/ps/nlin/0010050v1 
This figure "3.jpg" is available in "jpg" format from: http://arxiv.org/ps/nlin/0010050v1 
This figure "4.jpg" is available in "jpg" format from: http://arxiv.org/ps/nlin/0010050v1 
This figure "5.jpg" is available in "jpg" format from: http://arxiv.org/ps/nlin/0010050v1 
This figure "6.jpg" is available in "jpg" format from: http://arxiv.org/ps/nlin/0010050v1 
This figure "7a.jpg" is available in "jpg" format from: http://arxiv.org/ps/nlin/0010050v1 
This figure "7b.jpg" is available in "jpg" format from: http://arxiv.org/ps/nlin/0010050v1 
This figure "8.jpg" is available in "jpg" format from: http://arxiv.org/ps/nlin/0010050v1 